\title{
Remote-controlled deposit of superparamagnetic colloidal droplets
}

\author{
Alexis Darras, ${ }^{1,2,3, *}$ Florence Mignolet, ${ }^{1}$ Nicolas Vandewalle, ${ }^{1}$ and Geoffroy Lumay ${ }^{1}$ \\ ${ }^{1}$ GRASP, CESAM - Physics Department, University of Liège, B-4000 Liège; Belgium \\ ${ }^{2}$ F.R.S.-FRNS; B-1000 Bruxelles; Belgium \\ ${ }^{3}$ Experimental Physics, Saarland University; D-66123 Saarbrücken, Germany
}

(Dated: November 27, 2018)

\begin{abstract}
Evaporation of sessile droplets is a way to organize suspended particles and create surface coating. Many studies have demonstrated that suspensions with various composition can give rise to qualitatively different dried patterns, often by focusing on the radial density profile of deposited particles. We demonstrate that a single suspension of superparamagnetic colloids can give rise to several dried patterns thanks to an external magnetic field applied during the evaporation process. We show the various patterns obtained with zero, constant, rotating and oscillating magnetic fields, and evidence the continuous control given by the intensity of a constant magnetic field. We also show this magnetic control has a substantial effect on the morphological details of the deposits.
\end{abstract}

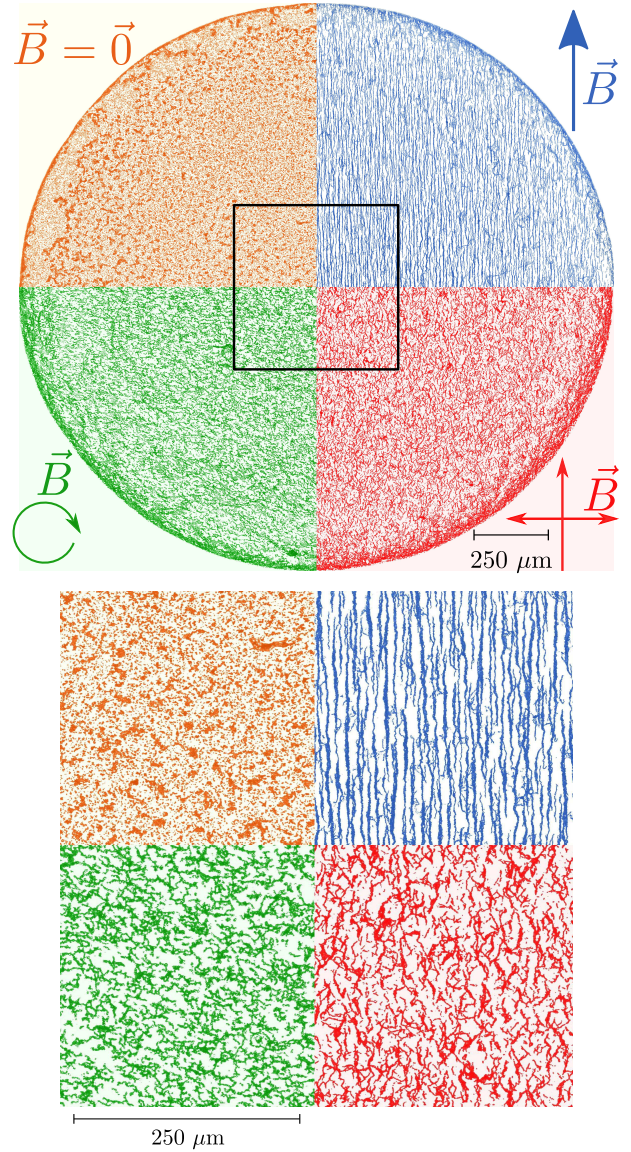

FIG. 1. Four deposits (four corners of the drop) obtained with different magnetic fields (null, homogeneous, rotating and oscillating fields, amplitude of non-zero magnetic fields are $22.5 \mathrm{G}$ ). Each picture has been numerically colorized, cropped as corner and positioned according to the corresponding field condition. The bottom picture is a zoom on the squared area. The global surface density of particles does not change very much. However, the morphological details of the deposit are radically different from one field to the other.

\section{INTRODUCTION}

The evaporation of colloidal droplets is currently a topic of intensive research due to its wide range of potential applications, extending from blood analysis $[1,2]$ to nanotechnology [3] via ink-jet printing, paint and polymers. [4] The main feature of colloidal droplets evaporation is the so-called coffee-ring effect.[5-9] This effect implies that particles tend to be deposited at the edge of the finally dried pattern. However, several studies have shown various mechanisms preventing this effect. Notably, capillary interactions of ellipsoidal particles [10] and the presence of surface-adsorbed polymers [11] allow a uniform coating of the particles. Marangoni flows created by temperature gradients, tensio-active agents or in binary mixtures can also strongly modify the deposit pattern.[11-15] More recently, some researchers have shown that more complex deposit structures can be achieved thanks to various concentrations of liquid crystals [16]. In this paper, we demonstrate that magnetic interactions between superparamagnetic colloidal particles [17-21] can be used to control the properties of colloidal droplets deposit, as illustrated in Fig.1. This actually requires the right chemical composition of the suspension. But then, a single suspension can result in various dried patterns. The actual pattern is selected from the application of an external magnetic field during the evaporation. This induces indeed a self-assembly of the particles[22-33] which is visible in the deposit. Following a quantitative description of the pattern obtained with homogeneous and constant field, we show that different patterns are produced by rotating and oscillating fields. To our knowledge, this is the first time that such a tunable control of evaporation deposit is described in the literature. 


\section{METHODS}

\section{Suspensions preparation}

Our experiments were performed with superparamagnetic microspheres (diameter $d=1.2 \mu \mathrm{m}$ ) dispersed in water (Estapor ${ }^{\circledR}$ M1-070/60), with a volume fraction of $\phi=510^{-4}$. Those particles are covered with carboxyl charged groups to enable electrostatic stabilization of the suspension. Further information and characterization on the particles can be found on the manufacturer's website [34]. Independent measurements were also performed and published in various scientific papers $[35,36]$. In order to clean the spheres from any chemical waste, the commercial suspensions are first deposited in the bottom of their container with a centrifuge. The supernatant is then removed and the same quantity of distilled water is then poured back into the container. The particles are then dispersed again in the liquid thanks to an ultrasonic bath and mechanical agitation. The whole process is repeated three times. After this cleaning process, the particles are deposited once again and the supernatant is replaced with the adequate solution, i.e. water with dilute phosphate buffered saline (PBS). The PBS has been chosen since it is a commercially available $\mathrm{pH}$ buffer involving only non-organic molecules. Indeed, $\mathrm{pH}$ is often used as a parameter to control electrostatic stabilization, and organic molecules could potentially feed bacteria who could reach the suspension as it ages. Particles are then ultra-sonicated again. After the ultrasonication, automatized size distribution measurements based on image analysis shows that the particles have the same mean diameter as in the original delivered suspension $(d=1.2 \mu \mathrm{m})$. This means that the particles are completely dispersed as corroborated by visual control of the suspensions observed with an optical microscope.

\section{Microscopy observations}

To perform the observations, a $2 \mu \mathrm{l}$ droplet of the suspension is placed on a microscope cover glass. A custom airtight evaporation chamber is placed upon it. The chamber is made of plexiglass and is T-shaped. The upper branches of the $\mathrm{T}$ are filled with silica gel to ensure reproducible humidity condition in the chamber. The central trunk is kept empty in order to keep a clear path for the light. Petroleum jelly is applied on the bottom of the chamber's base to assess airtightness between the plexiglass and the cover glass. A magnetic field $\vec{B}$ is applied by sending a current in surrounding coils at the beginning of each experiment. The magnetic field produced by those coils has been characterized with a Hall probe and, for a constant current, is homogeneous within the precision range of the probe of $2 \%$ around the cell. The constant current is sent in the coils with a constant intensity by a programmable DC power supply GenH750W from TDK Lambda, with a precision of $0.01 \mathrm{~A}$. The alternative currents producing rotating and oscillating fields are produced by a dual channel arbitrary function generator AFG3022C from Tektronix and amplified by a power amplifier TOE7610 from Toellner. The suspension is observed from the bottom with a $16 \mathrm{x}$ magnification. The microscope used is an inverted microscope Olympus IX73, connected to a 4070M-CL Thorlabs Camera with 2048 by 2048 pixels of 16 Bits depth.

\section{RESULTS}

We prepared a suspension of superparamagnetic colloids with a solid volume fraction of $\phi=5.10^{-4}$ in diluted PBS (volume fraction of PBS $\kappa=50.10^{-3}$ ). We then observed the evaporation of $2 \mu \mathrm{l}$ droplets under constant and homogeneous magnetic fields of intensity ranging from $0 \mathrm{G}$ to $22.5 \mathrm{G}$ by steps of $4.5 \mathrm{G}$. Other magnetic fields were also tested to determine the generality of our results, namely rotating and oscillating fields. For each of the specific conditions, we observed at least 5 different droplets evaporation in order to assess the reproducibility of the results.

Typical parts of eventually dried deposits are shown in Fig.1, for the various fields we tested. When there is no magnetic field, a slight coffee-ring trend is observed, but similar surface fractions of particles are also observed in the center of the deposit. This almost homogeneous deposition of particles is actually induced by solutal Marangoni flows, due to the presence of PBS, as described in a previous study. [13] However, when a magnetic field $|\vec{B}|$ is applied on the evaporating drop, the eventually dried deposit is radically different. The coffee-ring trend is even more reduced, as quantitatively described further, and structures resulting from the magnetic self-assembly of the particles are observed throughout the deposit. As we will show here in details for a constant and homogeneous magnetic field, the external field actually acts as a remote controller which can tune the morphological properties of the observed structures.

A quantitative analysis of the deposit can be performed by computing the mean surface density $\rho$ of the drop. We computed this quantity as a function of the relative distance from the center $r$ of the deposit, normalized by the radius $R$ of the deposit, for various amplitudes of a constant and homogeneous magnetic field $\vec{B}$. We then normalized this surface density $\rho$ by its integral $N=\int_{0}^{1} \rho(\delta) d \delta$, where $\delta=\frac{r}{R}$. This normalization enhances the relative variation values and ensures that no difference in lightening is taken into account. Resulting curves are presented in Fig.2. Those curves also enable to compute the ratio between the surface fraction in the middle of the drop $\rho(0) \equiv \rho_{m}$ and the surface fraction of 


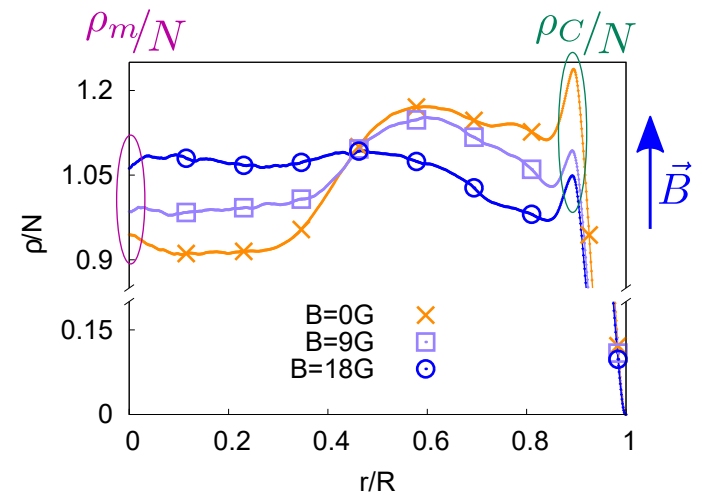

FIG. 2. Radial surface densities of the dried agglomerates normalized by the number of particles (so that the integral of the curve is equal to one), obtained with constant and homogeneous magnetic fields of various intensities. The density of particles is mainly uniform all over the drop, but a slight coffee-ring effect is observed as a local maximum near the edge. However, the amplitude of this peak decreases when the amplitude of the external magnetic field increases. The reference density values $\rho_{m}$ and $\rho_{C}$ used to quantify the coffee ring effect (see full text and Fig.3) are highlighted in this graph.

the particles in the coffee-ring $\rho_{C}$ (i.e. the value of the peak near the edge, see Fig.2). This ratio $\frac{\rho_{C}}{\rho_{m}}$ has been previously used to quantify the coffee-ring effect [10]. It is interesting to notice that for high magnetic fields, the ratio drops below 1, implying that the coffee ring somehow does not really exist in this case (see Fig.3). This affirmation has to be balanced by the fact that there is still a local maximum of the density near the edge of the deposit.

The mechanism through which the self-assembly influences the coffee-ring effect is by modifying the sedimentation time of the particles. Indeed, as demonstrated previously by Bhardwaj et al. [37], the transition from a coffee-ring to a homogeneous deposit can result from the competition between the sedimentation speed and the radial flow velocity. In their systems, the sedimentation speed is governed by a DLVO attraction between the particles and the substrate. The presence of PBS in our system ensures that we have such attraction too. However, the sedimentation speed also depends on the size of the particles through the gravitational sedimentation. Indeed, the gravitational sedimentation time $\tau_{s}$ over a typical dimension $a$ of the particles size is proportional to $\tau_{s} \propto \frac{\mathrm{Ca}}{\rho g a^{3}}$, where $\rho$ is the difference between the particles and the fluid density, $g$ is the acceleration of gravity and $C \propto \eta a$ is the drag coefficient depending on the viscosity $\eta$. The sedimentation time $\tau_{s}$ is then proportional to $\tau_{s} \propto a^{-1}$. On the other hand, since the magnetic field $\vec{B}$ induces the aggregation of the particles, this characteristic size $a$ also depends on this field amplitude $B$. More accurately, for short times, the chains grow with a Smoluchowsky law $\langle L\rangle=k\left(t / t_{B}\right)^{z}$, where $k$ is a geometric factor, $t_{B} \propto B^{-2}$ a characteristic time [23, 38] and $z$ is the kinetic exponent, usually measured between 0.6 and 0.7. [22-25]. If the characteristic length of the agglomerates is proportional to $\langle L\rangle \propto B^{\sim 1.3}$, then the sedimentation time is proportional to $\tau_{s} \propto B^{\sim-1.3}$. This then explains why self-assembly of particles lead to a decrease of the coffee-ring effect, depending on the magnetic field strength. Supplementary Materials contain videos of evaporating droplet to show the self-assembly process of the particles [39]. Consistently, similar results are obtained with oscillating and rotating fields, as illustrated in the Supplementary Materials [39].

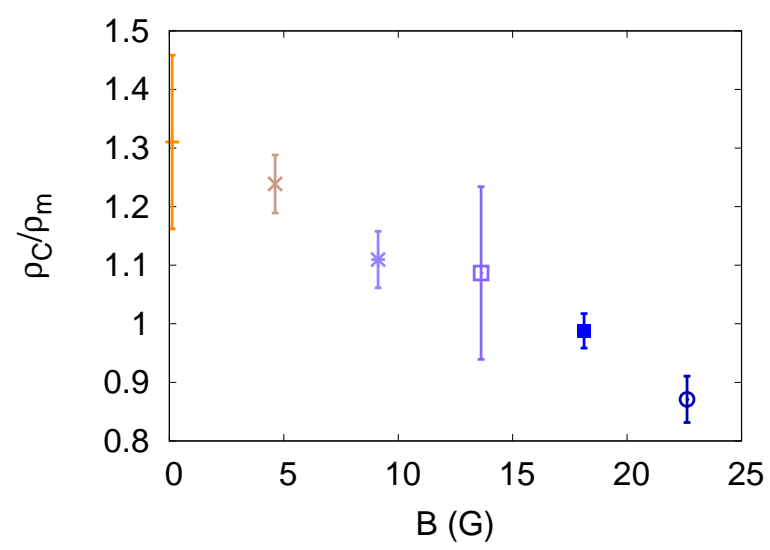

FIG. 3. Relative amplitude of the coffee rings as a function of the external magnetic field amplitude. This amplitude is computed as the ratio between the coffee ring density $\rho_{c}$ (value of the curves at the local maximum nearest from the drop's border), and the density in the center of the drop $\rho_{m}$. For high magnetic fields, this ratio drops below one, indicating that the coffee-ring effect is mainly countered. The deposit is then almost uniform.

In our experiment, the magnetic field also changes morphological properties of the deposit. Namely, for a constant and homogeneous magnetic field, the chains formed by the particles during the evaporation process remain in the deposit. It is worthwhile to notice that in the case of lower Marangoni stress, as obtained with lower concentration of PBS, those structures do not necessarily deposits this way (see video in [39]). However, in the case of sufficient PBS concentration, this morphological change can be assessed by computing the local major-axis length $L$ of the disjoint parts of the finally dried deposit (i.e. the disjoint agglomerates constituting the dried deposit). In the case of a constant field $\vec{B}$, the agglomerates are chains of particles aligned with the external magnetic field. The typical size of those agglomerates varies with the magnetic field intensity, but except at the edge of the deposit, it does not strongly depend on the position in the deposit, as illustrated in Fig.4 for the constant magnetic field. Those chains become longer as the magnetic field increases and the chains are observed everywhere in 


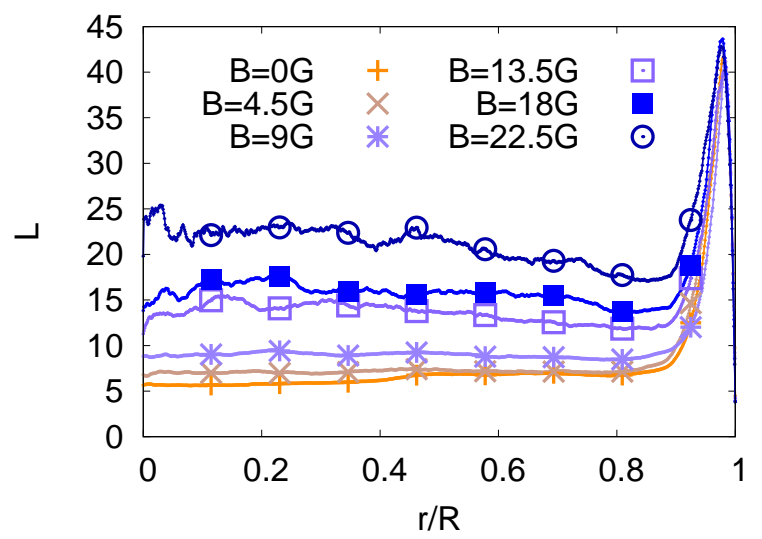

FIG. 4. Evolution of the mean major-axis length of the dried agglomerates (in particle's diameter), as a function of the normalized radial distance $r / R$. The main peak near the edge of the drop is actually caused by the coffee ring, which is locally seen as a very long agglomerate of particles. However, this length does not strongly depend on the position elsewhere and globally increases with the external magnetic field.

the finally dried deposit. The global averaged length of the chains all over the droplet increases with the amplitude of the magnetic field as can be seen in Fig.5. The influence of the magnetic field on this parameter of the deposit can be modeled by assuming that the chains are self-organized as in an airtight chamber of liquid and then abruptly stopped after a constant time $t_{e}$ which can be assimilated to the evaporation time of the droplet. Indeed, given the Smoluchowsky law $\langle L\rangle=k\left(t / t_{B}\right)^{z}$, at the time $t_{e}$ where the evaporation of the droplet stops the assembly of the chains, the mean length of the particles should be $\langle L\rangle=k\left(t_{e} / t_{B}\right)^{z} \propto B^{2 z}$. However, in our experiments, Marangoni flows can create particles agglomerates, initially at the top surface of the droplet, even without magnetic field. A first approach to take this agglomeration mechanism into account is to assume they simply shift this mean length and then consider a fit $\langle L\rangle=a B^{2 z}+\langle L\rangle_{0}$, with $a$ and $\langle L\rangle_{0}$ being two fitting parameters, where $a$ takes into account the evaporation time and the properties of both the fluid and the particles, while $\langle L\rangle_{0}$ is the shift created by the Marangoni recirculation. By fixing the kinetic exponent to the typical value $z=0.65$, we obtain the curve in Fig.5, whose coefficient of determination $R^{2}$ is about $R^{2}=0.97$. The power-law behavior of the data is enhanced in this Fig.5 by an appropriate scaling. The good agreement of this simple model with the data shows that the dominant mechanism determining the properties of the deposit in our system is indeed the magnetic self-assembly of the particles. This observation is reinforced by the various eventually dried deposits observed with different magnetic field configurations as illustrated in Fig.1. To obtain those deposits, we used magnetic fields of $22.5 \mathrm{G}$

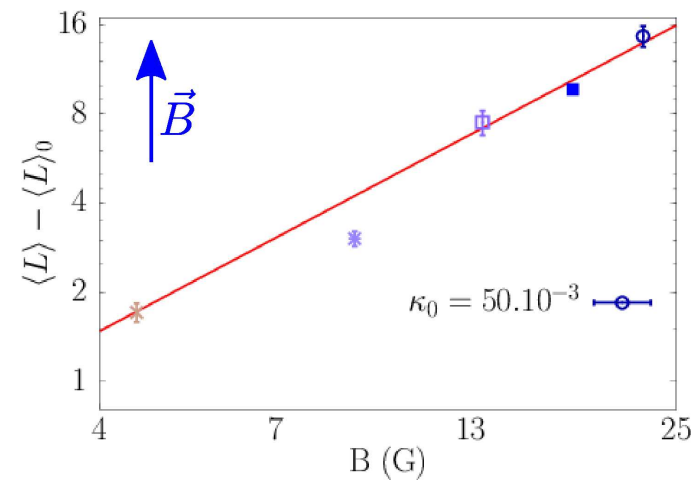

FIG. 5. Global averaged value of the major-axis length of the agglomerates in the deposit. This mean length $\langle L\rangle$ is expressed as a function of the external magnetic field amplitude. The dots are experimental data, and the curve is a fit of the model $\langle L\rangle=a B^{2 z}+\langle L\rangle_{0}$ explained in the full text. The coefficient of determination $R^{2}$ of the fit is $R^{2}=0.97$. In order to enhance the power-law behavior of the model, we subtracted the zero-field aggregate size $\langle L\rangle_{0}$.

amplitude, and oscillating currents that have a frequency of $50 \mathrm{~Hz}$. Rotating fields are generated with a sine current in the coils creating the field along the X-axis, and a cosine current in the coils creating the field along the $\mathrm{Y}$ axis. The sign of the cosine is reversed every turn in order to avoid rotation of the particles [29]. The oscillating field is generated with a constant magnetic field along the $\mathrm{Y}$-axis and a sine-shape field along the $\mathrm{X}$-axis. While the global surface density distribution of the particles is comparable between the four conditions (zero, constant, rotating and oscillating field), the morphological details of the deposit are radically different, as expected from the previous observations. Rotating fields create deposits with connected branches without preferential direction, as expected from previous studies. [29] Under oscillating fields, the final structures still have a preferential orientation along the average direction of the field. However, they locally acquire deviations which break the chains and connect them to their neighbors, creating a pattern in-between the cases of constant and rotating fields.

\section{CONCLUSION}

Those experiments show that, with the right strength of solutal-Marangoni instabilities, an external magnetic field can be used as a remote control of the eventually dried deposit. Indeed, due to this field, superparamagnetic colloids self-organize into structures that can be deposited almost as is on the substrate. This fine tunable control of dried deposits open new ways to implement promising applications in micro-fabrication employing textured coatings (e.g., see [40-42]). Moreover, since the parameter responsible for the self-organization of the particles is the magnetic field, and given magnetic in- 
teractions can be easily written in equations, this experimental setup can be used as a model system to understand more complex systems. For instance, blood or protein deposits are known to give rise to peculiar patterns [43-45]. However, their interactions is quite complex. Tunable interactions from our set-up might then be a good starting point to investigate how interactions between solid component influences their deposits.

\section{CONFLICTS OF INTEREST}

There are no conflicts to declare.

\section{ACKNOWLEDGEMENTS}

A.D. is financially supported by FNRS as research fellow. This work was financially supported by the FNRS (Grant PDR T.0043.14) and by the University of Liège (Starting Grant C-13/88).

* alexis.darras@ulg.ac.be

[1] T. A. Yakhno, V. G. Yakhno, A. G. Sanin, O. A. Sanina, A. S. Pelyushenko, N. A. Egorova, I. G. Terentiev, S. V. Smetanina, O. V. Korochkina, and E. V. Yashukova, IEEE engineering in medicine and biology magazine $\mathbf{2 4}$, 96 (2005).

[2] D. Brutin, B. Sobac, B. Loquet, and J. Sampol, Journal of Fluid Mechanics 667, 85 (2011).

[3] H. Y. Erbil, Advances in colloid and interface science 170, 67 (2012).

[4] K. Sefiane, Advances in colloid and interface science 206, 372 (2014).

[5] R. D. Deegan, O. Bakajin, T. F. Dupont, G. Huber, et al., Nature 389, 827 (1997).

[6] R. D. Deegan, O. Bakajin, T. F. Dupont, G. Huber, S. R. Nagel, and T. A. Witten, Physical review E 62, 756 (2000).

[7] A. G. Marín, H. Gelderblom, D. Lohse, and J. H. Snoeijer, Phys. Rev. Lett. 107, 085502 (2011).

[8] Á. G. Marín, H. Gelderblom, D. Lohse, and J. H. Snoeijer, Physics of fluids 23, 091111 (2011).

[9] Q. Xie and J. Harting, Langmuir 34, 5303 (2018).

[10] P. J. Yunker, T. Still, M. A. Lohr, and A. Yodh, Nature 476, 308 (2011).

[11] H. Kim, F. Boulogne, E. Um, I. Jacobi, E. Button, and H. A. Stone, Physical review letters 116, 124501 (2016).

[12] H. Hu and R. G. Larson, The Journal of Physical Chemistry B 110, 7090 (2006).

[13] A. Darras, N. Vandewalle, and G. Lumay, Phys. Rev. E. , accepted for publication (2018).

[14] R. Bennacer and K. Sefiane, Journal of Fluid Mechanics 749, 649 (2014).

[15] J. R. Christy, Y. Hamamoto, and K. Sefiane, Physical review letters 106, 205701 (2011).
[16] Z. S. Davidson, Y. Huang, A. Gross, A. Martinez, T. Still, C. Zhou, P. J. Collings, R. D. Kamien, and A. Yodh, Nature Communications 8, 15642 (2017).

[17] J. Faraudo, J. S. Andreu, C. Calero, and J. Camacho, Advanced Functional Materials 26, 3837 (2016).

[18] J. W. Tavacoli, P. Bauër, M. Fermigier, D. Bartolo, J. Heuvingh, and O. du Roure, Soft Matter 9, 9103 (2013).

[19] W. Wen, L. Zhang, and P. Sheng, Physical review letters 85, 5464 (2000).

[20] X. Xu, G. Friedman, K. D. Humfeld, S. A. Majetich, and S. A. Asher, Advanced materials 13, 1681 (2001).

[21] A. Snezhko and I. S. Aranson, Nature materials 10, 698 (2011).

[22] J. S. Andreu, J. Camacho, and J. Faraudo, Soft Matter 7, 2336 (2011).

[23] J. H. Promislow, A. P. Gast, and M. Fermigier, J. Chem. Phys. 102, 5492 (1995).

[24] P. Domínguez-García, J. Pastor, and M. Rubio, Eur. Phys. J. E 34, 1 (2011).

[25] P. Domínguez-García, S. Melle, J. Pastor, and M. Rubio, Phys. Rev. E 76, 051403 (2007).

[26] M. Fermigier and A. P. Gast, Journal of colloid and interface science 154, 522 (1992).

[27] A. Darras, J. Fiscina, M. Pakpour, N. Vandewalle, and G. Lumay, The European Physical Journal E 39, 1 (2016).

[28] A. Darras, J. Fiscina, N. Vandewalle, and G. Lumay, American Journal of Physics 85, 265 (2017).

[29] K. Müller, N. Osterman, D. Babič, C. N. Likos, J. Dobnikar, and A. Nikoubashman, Langmuir 30, 5088 (2014).

[30] M. Llera, J. Codnia, and G. A. Jorge, J. Magn. Magn. Matter 384, 93 (2015).

[31] R. M. Erb, H. S. Son, B. Samanta, V. M. Rotello, and B. B. Yellen, Nature 457, 999 (2009).

[32] H. Carstensen, V. Kapaklis, and M. Wolff, Phys. Rev. E 92, 012303 (2015).

[33] K. V. Edmond, H. Park, M. T. Elsesser, G. L. Hunter, D. J. Pine, and E. R. Weeks, Chaos-Woodbury 21, 041103 (2011).

[34] S. D. Sheets and A. Finder, "Polymeric microspheres and magnetic beads," http://merckmillipore.com/.

[35] P. Domínguez-García, S. Melle, J. Pastor, and M. Rubio, Physical Review E 76, 051403 (2007).

[36] M. Fermigier and A. P. Gast, Journal of Colloid and Interface Science 154, 522 (1992).

[37] R. Bhardwaj, X. Fang, P. Somasundaran, and D. Attinger, Langmuir 26, 7833 (2010).

[38] A. Darras, E. Opsomer, N. Vandewalle, and G. Lumay, Scientific reports 7, 7778 (2017).

[39] [See supplementary materials at [URL will be inserted by Editor] for rotating and oscillating magnetic fields data and illustrative videos of the droplets evaporation.].

[40] R. A. Bidkar, A. J. Kulkarni, L. S. Leblanc, and V. Bahadur, "Reduced fluid drag across a solid surface with a textured coating," (2015), uS Patent App. 14/037,790.

[41] K. P. Gadkaree, F. M. Joos, J. R. Lim, K. P. Reddy, and J. E. Tosch, "Current collectors having textured coating," (2015), uS Patent 9,209,464.

[42] T. Koshi and H. Kondo, "Waterproof, unevenly textured coating film," (1988), uS Patent 4,761,312.

[43] L. Lanotte, D. Laux, B. Charlot, and M. Abkarian, Phys. Rev. E 96, 053114 (2017). 
[44] T. Yakhno, O. Sedova, A. Sanin, and A. Pelyushenko, Techn. Phys. 48, 399 (2003).

[45] G. Chen and G. J. Mohamed, The European Phys. J. E 33, 19 (2010). 